\title{
Association of tribbles homologue 1 gene expression in human umbilical vein endothelial cells with duration of intrauterine exposure to hyperglycaemia
}

\author{
POLINA V. POPOVA ${ }^{1,2 *}$, LIUDMILA B. VASILEVA ${ }^{1}$, ALEXANDRA S. TKACHUK $^{1}$, \\ MAXIM V. PUZANOV ${ }^{1}$, YANA A. BOLOTKO ${ }^{1}$, EVGENII A. PUSTOZEROV ${ }^{1,3}$, ANDREY \\ S. GERASIMOV ${ }^{1}$, IRINA E. ZAZERSKAYA ${ }^{1}$, OLGA A. LI ${ }^{1}$, ELENA Y. VASILYEVA ${ }^{1}$, \\ ANNA A. KOSTAREVA ${ }^{1}$, RENATA I. DMITRIEVA ${ }^{1}$ AND ELENA N. GRINEVA ${ }^{1,2}$ \\ ${ }^{1}$ Almazov National Medical Research Centre, Saint Petersburg, Russian Federation \\ ${ }^{2}$ Department of Internal Diseases and Endocrinology, St Petersburg Pavlov State Medical University, Saint Petersburg, Russian Federation \\ Saint Petersburg State Electrotechnical University, Saint Petersburg, Russian Federation
}

(Received 19 November 2017; revised 23 January 2018; accepted 6 February 2018)

\section{Summary}

Maternal gestational diabetes mellitus (GDM) is considered to be an important factor that epigenetically predisposes offspring to metabolic and cardiovascular diseases. However, the mechanisms of how intrauterine hyperglycaemia affects offspring have not been thoroughly studied. The mammalian tribbles homologue 1 (TRIB1) gene is associated with plasma lipid concentrations and coronary artery disease (CAD). Our aim was to study the effect of GDM and its treatment terms on the level of TRIBI gene expression in human umbilical vein endothelial cells (HUVECs) of newborns from women with and without GDM. The study included 50 women with GDM and 25 women without GDM (control group). Women with GDM were divided into three groups according to their gestational age when the treatment of GDM started: 24-28 weeks (GDM1, $\mathrm{N}=16), 29-32$ weeks (GDM2, $\mathrm{N}=25)$ and $>34$ weeks (GDM3, $\mathrm{N}=9$ ). The levels of TRIB1 gene expression in GDM3, GDM2, GDM1 and control groups were $2.8 \pm 1.1,4.2 \pm 2.4,6.0 \pm 3.4$ and $8.1 \pm 6.1$, respectively $(p$ $=0.001)$. After comparison in pairs the difference was significant for the following pairs: GDM2-control $(p=$ $0.004)$, GDM3-control $(p=0.002)$, GDM1-GDM3 ( $p=0.012)$. Notably, if treatment had been started before the 28th week of gestation, the difference in TRIBI gene expression in HUVECs was not significant $(p=0.320$ for comparison between GDM1 and control groups). Our findings support the hypothesis that TRIB1 gene expression in HUVECs depends on the duration of intrauterine exposure to hyperglycaemia.

\section{Introduction}

Gestational diabetes mellitus (GDM) is the most common complication of pregnancy affecting $17.8 \%$ (range 9.3-25.5\%) of pregnancies according to the criteria of the International Association of Diabetes and Pregnancy Study Groups (IADPSG) (Sacks et al., 2012). Apart from the short-term complications (HAPO Study Cooperative Research Group et al., 2008) and long-term problems for the mother, GDM is also associated with the development of type 2 diabetes, obesity and cardiovascular diseases in offspring (Ma et al., 2015; Damm et al., 2016). The studies reporting the association of the above conditions

\footnotetext{
* Corresponding author: Institution of Endocrinology, Almazov National Medical Research Centre, 2 Akkuratov St, 197341, Saint Petersburg, Russian Federation. E-mail: pvpopova@yandex.ru
}

with intrauterine hyperglycaemia support the fetal programming (Barker's) and the Developmental Origins of Health and Diseases (DOHaD) hypotheses (Barker, 1999; Pettitt et al., 2008; Tam et al., 2010; Nielsen et al., 2012). It is crucial to look into the mechanisms by means of which maternal gestational diabetes affects the development of cardiovascular diseases among offspring, in order to work out effective measures for prenatal prevention. According to a number of studies, GDM is associated with altered methylation patterns and expression of genes involved in the pathogenesis of metabolic diseases (Bouchard et al., 2010; Bouchard et al., 2012; Ruchat et al., 2013). However, association does not confirm causation.

It could just as well be the opposite: that the changes in gene methylation cause hyperglycaemia in the mother, in which case the genes regulating insulin resistance or insulin secretion are involved and the 
methylation pattern of these genes is transferred to the offspring. We could also suppose that another pathological process is the cause of both maternal hyperglycaemia and epigenetic changes in the genome of the fetus.

Studies comparing the achieved glucose levels during the treatment of GDM with the changes in levels of methylation and expression of target genes in newborns could help define the cause-and-effect relationship.

Another plausible way to test the causal role of intrauterine hyperglycaemia is to compare the levels of expression of target genes in newborns from women with different durations of hyperglycaemia during pregnancy. Normally, there is a relatively narrow timeframe when screening for GDM is recommended (24-28 weeks of gestation). However, real clinical practice sometimes provides the opportunity to include those women who missed the recommended timeframe for oral glucose tolerance test (OGTT) or delayed their referral to healthcare providers after OGTT and therefore were not diagnosed with GDM in due time. It is logical to presume that such women have a longer duration of hyperglycaemia compared to those with GDM diagnosed in a timely manner and effectively treated.

Among candidate genes associated with cardiovascular diseases and lipid homeostasis the tribbles gene family deserve particular attention as its role was confirmed by both genetics and experimental biology (Angyal \& Kiss-Toth, 2012). Several studies have reported an association of variants of tribbles homologue 1 (TRIBI) with plasma lipid concentrations (triglycerides, highdensity lipoprotein cholesterol [HDL-C] and cholesterol) (Willer et al., 2008; Nakayama et al., 2009; Edmondson et al., 2011) and with an increased risk of ischemic heart disease (Willer et al., 2008; Tai et al., 2009; Varbo et al., 2010). In animal studies the experimental evidence of a controlling role of TRIB1 in serum lipid levels was provided by the study by Burkhardt et al. (2010). The hepatic overexpression of TRIB1 reduced very-low-density lipoprotein (VLDL) production, lowering plasma triglyceride and cholesterol levels. By contrast, in TRIB1 knockout mice an increase in VLDL production and a consequent rise in triglyceride and cholesterol levels were observed (Burkhardt et al., 2010).

Our aim was to study the effect of GDM and its treatment terms on the level of TRIBI gene expression in human umbilical vein endothelial cells (HUVECs) of newborns from women with and without GDM.

\section{Materials and methods}

(i) Study participants

This study included 75 pregnant women. Fifty women with GDM were divided into three groups according to gestational age when their treatment for GDM started: 24-28 weeks (GDM1, N = 16), 29-32 weeks $($ GDM2, N $=25)$ and $\geq 34$ weeks $($ GDM3,$N=9)$. All women received an OGTT between 24-31 weeks of gestation. Nine women who visited their physicians to receive the results of OGTT and thus classified as having GDM only during or after the 34th week were available in our cohort and were therefore selected for the group with late diagnosis (GDM3). Twenty five women with normal glucose tolerance comprised the control group. The study was approved by the local ethics committee of Almazov National Medical Research Center (ANMRC; Protocol 15-119). All participants provided written informed consent.

GDM was diagnosed according to the criteria of the Russian national consensus (Dedov et al., 2012) and the IADPSGs Consensus Panel (International Association of Diabetes Pregnancy Study Groups Consensus Panel, 2010) based on the results of $2 \mathrm{~h}$ OGTT performed between 24-31 weeks of gestation.

Women with GDM were included in the GDM1 group if their gestational age at the time of inclusion was 24-28 weeks and were allocated to the GDM2 group if it was 29-32 weeks at the time of inclusion. The reasons for the late start of GDM treatment were late communication of OGTT results or delay in referral to endocrinologists. Women in the control group were included at any time of pregnancy after OGTT ( $>24$ weeks).

Exclusion criteria were preexisting diabetes mellitus, identified fetal anomalies, nonsingleton pregnancies, and conditions or use of drugs affecting carbohydrate metabolism.

All participants were followed until delivery at ANMRC. Women with GDM visited the endocrinologists every 1-3 weeks and provided the results of their self measurements of blood glucose (BG). The target glycemic goals for women with GDM were to keep the fasting $\mathrm{BG}<5.3 \mathrm{mmol} / \mathrm{l}$ and $1 \mathrm{~h}$ postprandial $<7.8 \mathrm{mmol} / \mathrm{l}$. In addition, all participants were encouraged to keep electronic nutrition and glycemic control diaries with the help of a specially developed mobile application and send data to the doctor. Women with GDM were recommended to do it during the whole period of the study, while the control group were asked to do it only during the first week after inclusion and in the 36th week of gestation (if included before the 36th week). According to the personal diaries, automatic calculations of the integral indicators characterizing the self-control of glycaemia (fasting, postprandial and average glycaemia) were accomplished.

According to local delivery protocol, the timing of delivery for women with GDM was planned based on the results of the ultrasound examination performed between 36-37 weeks of gestation. Women with normal estimated fetal weight were managed expectantly until the 40th week. If the estimated 
fetal weight exceeded the 90th percentile labour was induced at 37-39 weeks. Labour was also induced for non-GDM related maternal or fetal indications, for instance preeclampsia.

\section{(ii) Blood samples and analyses}

Fasting maternal blood samples were collected at the time of inclusion in the study. The umbilical cord blood samples were taken from one of the umbilical arteries immediately after delivery. Maternal and cord blood samples were centrifuged and aliquots of serum were stored at $-80^{\circ} \mathrm{C}$ until analysis.

Plasma glucose (PG) concentration was determined by the glucose oxidase method. Total cholesterol, HDL-C, LDL-C, VLDL-C and triglyceride levels were measured through enzymatic colorimetric methods with a diagnostic reagent system for the Cobas Integra Autoanalyzer. Serum C-peptide level was measured by the immunoassay method (Abbott Diagnostics).

\section{(iii) Isolation and identification of HUVECs}

The isolation of the HUVECs was performed using a standard collagenase digestion method (Jaffe et al., 1973). The cells were cultured and expanded in Endothelial Cell Medium (ECM; ScienCell) containing $5 \%$ fetal bovine serum, $1 \%$ penicillin/streptomycin and Endothelial Cell Growth Supplement (ScienCell Cat $\# 1052$ ). Cultivation of cell cultures was performed in a $\mathrm{CO}_{2}$ incubator with $5 \%$ carbon dioxide at $+37^{\circ} \mathrm{C}$. The medium was changed after $24 \mathrm{~h}$ and every 2 days thereafter until confluence. After the expansion of the required number of cells (about 4-6 million), they were frozen for long-term storage in liquid nitrogen. For this study, HUVECs at passages $2-3$ were used. The flow cytometry analysis was carried out on Guava EasyCyte8 to evaluate the purity of primary HUVEC cultures.

Isolated cells were resuspended in $200 \mu \mathrm{l}$ of PBS containing 1\% BSA (Sigma-Aldrich) and incubated for $15 \mathrm{~min}$ at $20^{\circ} \mathrm{C}$ with the following antibodies $(\mathrm{Ab})$ : FITC-conjugated anti-CD31 (BioLegend), PE-Aconjugated anti-CD144 (BioLegend), PE-A-conjugated anti-CD105 (Bioscience Pharmingen), PE-Cy7-Aconjugated anti-CD146 (BioLegend) and APC-Aconjugated anti-CD45 (DAKO). The data were processed using the FACSDiva software program (version 6.1.3; BD Biosciences).

Counting was carried out when the threshold value of the number of events reached 7000 in one sample. As an isotypic (negative) control, cultures of the same uncoloured cells were used.

\section{(iv) Evaluation of apoptosis}

The viability of HUVECs was assessed by flow cytometry with the determination of the number of viable cells, as well as those in early, late apoptosis and necrosis evaluated by Annexin-V/PI (BioLegend) double staining. Sample preparation and dyeing were carried out in accordance with the recommendations of the manufacturers.

\section{(v) Immunocytochemical assay}

The procedure of immunocytochemical staining was used to assess the expression of von Willebrand factor (vWF) and CD146 (BioLegend) in HUVECs. HUVEC cultures, seeded onto cover glasses, were fixed with $4 \%$ paraformaldehyde. Fixed samples were then treated with mouse antibody against human vWF and stained with the fluorescent dye Alexa-546 conjugated with anti-mouse antibody. The samples were also treated with rabbit antibody against human CD146 and stained with the fluorescent dye Alexa-488 conjugated with anti-rabbit antibody. The vWF and CD146 expression was analysed by fluorescent microscopy. Cell nuclei were stained with DAPI.

\section{(vi) $R T-q P C R$}

Total RNA was extracted from HUVECs using ExtractRNA (BC032, Evrogen) according to the manufacturer's protocol. RNA concentration was calculated using a Nanodrop ND1000 spectrophotometer (NanoDrop Technologies). RNA was reverse transcribed using the MMLV RT Kit (SK021, Evrogen) according to manufacturer's instructions. Gene expression was evaluated by real-time PCR using Applied Biosystems TaqMan Gene Expression Assays which consist of a pair of unlabeled PCR primers and a TaqMan probe and normalized to the levels of GAPDH mRNA. Relative expression between a given sample and a reference sample was calculated according to the $2^{-\Delta \Delta \mathrm{Ct}}$ method (Livak \& Schmittgen, 2001).

\section{(vii) Statistical analysis}

Statistical analysis was performed using the GraphPad Instat computer program (GraphPad Software) and SPSS 22.0 (SPSS Inc., USA). Continuous variables were presented as mean and standard deviation. Numbers and percentages were reported for categorical variables. Differences among the groups were analysed by Mann-Whitney test (for comparison between two groups), Kruskal-Wallis test (for comparison of more than two groups) or Chi-square tests. Spearman's correlation coefficient was used to assess the association between parameters of glucose control and the level of expression of the TRIBI gene. A $p$-value $<0.05$ was considered statistically significant. 


\section{Results}

\section{(i) Characteristics of the participants}

Baseline characteristics of the participants are presented in Table 1. There was no difference among the groups in age and prepregnancy body mass index (BMI). According to the inclusion criteria, the women in the GDM2 and GDM3 groups were included in the study at significantly higher gestational age compared to GDM1, $p<0.001$ for both comparisons. Systolic and diastolic blood pressure (BP) at the time of study entry was higher in the GDM2 and GDM3 groups compared to the control group. Systolic BP was also higher in the GDM3 vs. GDM1 group ( $p=0.043)$.

The three GDM groups had higher levels of PG in OGTT compared to controls with no difference among the GDM groups. Serum lipid levels did not differ significantly except for HDL-C, which was lower in the GDM3 group compared to all other studied groups.

\section{(ii) Parameters of metabolic control and electronic diaries}

The metabolic control parameters and the results of the electronic diaries during GDM treatment are presented in Table 2. Gestational weight gain was significantly lower in the GDM1 and GDM2 groups compared to the control group $(p=0.007$ and $p=0.036$, respectively) and did not differ between the GDM3 and control groups. The level of systolic BP measured within one week of delivery was higher in the GDM3 group than in the control group $(p=0.008)$ and GDM2 group $(p=0.004)$. The level of diastolic BP was higher in the GDM1 $(p=0.005)$ and GDM2 groups $(p=0.003)$ compared with the control group.

The levels of average, fasting and postprandial glycaemia measured by participants during the study did not significantly differ among the four groups ( $p=0.051,0.592$ and 0.078 , respectively). Although, the average and postprandial BG levels tended to be lower in the GDM1 group compared to the GDM2 and control groups.

The percentages of women treated with insulin were 31,40 and $33 \%$ in the GDM1, GDM2 and GDM3 groups, respectively, and did not significantly differ $(p=0.836)$.

\section{(iii) Pregnancy outcomes}

Pregnancy outcomes and biochemical parameters in cord blood are presented in Table 3. There was no substantial difference among the groups in gestational age at delivery, birthweight, as well as in the levels of glucose, C-peptide and lipids in cord blood serum.
The differences in the frequencies of caesarean section and small for gestational age (SGA) newborns were also not significant, presumably due to the small sample size. The frequency of large for gestational age (LGA) newborns was increased with increased gestational age at the time of GDM treatment start $(p=0.033)$. However, after comparison in pairs only the difference between the GDM3 and the control group was significant $(p=0.017)$.

\section{(iv) HUVECs characterization}

HUVECs demonstrated characteristic endothelial morphology and immunophenotype: CD45 APC-/ CD144 PE+/CD31 FITC+/CD146 PE/Cy7+/CD105 $\mathrm{PE}+$ in all groups. Immunocytochemical staining of endothelial markers of VWF and CD146 were detected in all populations, which confirms the endothelial nature of these cells (Fig. 1).

There was no difference in the parameters of viability and replicative aging of HUVEC cultures from different patient groups.

(v) TRIB1 gene expression in HUVECs and correlation with maternal clinical parameters

The levels of TRIB1 gene expression in the GDM3, GDM2, GDM1 and the control group were $2.8 \pm 1.1$, $4.2 \pm 2.4,6.0 \pm 3.4$ and $8.1 \pm 6.1$, respectively $(p=0.001)$. After comparison in pairs the difference was significant for the following pairs: GDM2-control $(p=0.004)$, GDM3-control $(p=0.002)$ and GDM1-GDM3 $(p=$ 0.012) (Fig. 2). Notably, down regulation of TRIBI gene expression in HUVECs of GDM mothers was gone if treatment had been started before the 28th week of gestation ( $p=0.320$ for comparison between GDM1 and the control group). Some negative correlations have been observed between the level of TRIB1 gene expression and the following parameters: gestational age when treatment for GDM started $(\mathrm{r}=-0.417, p=0.003)$; plasma glucose levels $1 \mathrm{~h}$ and $2 \mathrm{~h}$ in OGTT $(\mathrm{r}=-0.381, p=0.002$ and $\mathrm{r}=-0.368, p=0.003$, respectively) and with SGA state $(\mathrm{r}=-0.327, p=0.005)$. Notably, there was no correlation of the level of TRIBI gene expression with gestational weight gain, systolic and diastolic BP measured within one week of delivery (data not presented) and maternal serum HDL-C levels, despite the difference in these parameters between the control and GDM groups. If only women with GDM were included in the analysis (without the control group), there was no correlation of TRIBI gene expression with plasma glucose levels $1 \mathrm{~h}(p=0.270)$ and $2 \mathrm{~h}$ in OGTT $(p=0.260)$, and with average $(p=0.118)$, fasting $(p=0.686)$ and postprandial glycaemia $(p=0.249)$ measured by the participants during the study. 
Table 1. Clinical and anthropometric characteristics of the participants.

\begin{tabular}{|c|c|c|c|c|c|}
\hline & $\begin{array}{l}\text { GDM1 } \\
(\mathrm{N}=16)\end{array}$ & $\begin{array}{l}\text { GDM2 } \\
(\mathrm{N}=25)\end{array}$ & $\begin{array}{l}\text { GDM3 } \\
(\mathrm{N}=9)\end{array}$ & $\begin{array}{l}\text { Control } \\
(\mathrm{N}=25)\end{array}$ & $\begin{array}{l}\text { Overall } \\
P \text {-value }\end{array}$ \\
\hline Maternal age, years & $32.6 \pm 5.9$ & $30.8 \pm 4.6$ & $32.7 \pm 3.8$ & $30.8 \pm 4.1$ & 0.532 \\
\hline Gestational age at study entry, weeks & $26.3 \pm 1.6^{*}$ & $30.5 \pm 1.0^{\&}$ & $36.0 \pm 1.9^{*, \& \&, \$ \$}$ & $30.2 \pm 4.3$ & $<0.001$ \\
\hline Prepregnancy BMI, $\mathrm{kg} / \mathrm{m}^{2}$ & $26.9 \pm 6.4$ & $25.0 \pm 7.0$ & $29.6 \pm 10.9$ & $23.4 \pm 4.2$ & 0.194 \\
\hline $\mathrm{BP}$ syst, $\mathrm{mmHg}$ & $117 \pm 13$ & $121 \pm 13^{*}$ & $133 \pm 21^{*, \&}$ & $112 \pm 14$ & 0.014 \\
\hline $\mathrm{BP}$ diast, $\mathrm{mmHg}$ & $74 \pm 11$ & $75 \pm 9 *$ & $82 \pm 10^{*}$ & $69 \pm 8$ & 0.007 \\
\hline Fasting $\mathrm{PG}, \mathrm{mmol} / 1$ & $5.0 \pm 0.6^{*}$ & $5.0 \pm 0.7 *$ & $5.3 \pm 0.7^{*}$ & $4.5 \pm 0.4$ & 0.002 \\
\hline OGTT 1-h PG, mmol/l & $9.8 \pm 1.5 * *$ & $10.2 \pm 1.5^{* *}$ & $10.7 \pm 1.8 * *$ & $6.9 \pm 1.9$ & $<0.001$ \\
\hline OGTT 2-h PG, mmol/1 & $8.1 \pm 1.2 * *$ & $8.6 \pm 1.9 * *$ & $8.8 \pm 1.4^{* *}$ & $5.9 \pm 1.5$ & $<0.001$ \\
\hline Total cholesterol (mmol/l) & $6.8 \pm 1.2$ & $6.8 \pm 1.3$ & $5.9 \pm 0.3$ & $6.1 \pm 1.1$ & 0.058 \\
\hline Triglycerides (mmol/l) & $1.9 \pm 0.6$ & $2.2 \pm 0.9$ & $2.6 \pm 0.9$ & $1.9 \pm 0.9$ & 0.131 \\
\hline $\mathrm{HDL}-\mathrm{C}(\mathrm{mmol} / \mathrm{l})$ & $2.0 \pm 0.5$ & $2.0 \pm 0.3$ & $1.6 \pm 0.4^{*, \&, \$}$ & $1.9 \pm 0.4$ & 0.042 \\
\hline LDL-C (mmol/l) & $3.9 \pm 1.1$ & $3.7 \pm 1.0$ & $3.1 \pm 0.6$ & $3.3 \pm 0.9$ & 0.240 \\
\hline
\end{tabular}

BMI: body mass index; BP: blood pressure; HDL-C: high-density lipoprotein-cholesterol; LDL-C: low-density lipoprotein cholesterol; OGTT: oral glucose tolerance test; PG: plasma glucose.

$*, * * P<0.05,<0.01$ vs. control.

$\stackrel{\& \& \&}{ } P<0.05,<0.01$ vs. GDM1

${ }^{\$, \$} P<0.05,<0.01$ vs. GDM2.

Table 2. Parameters of metabolic control and electronic diaries.

\begin{tabular}{llllll}
\hline \hline & GDM1 $(\mathrm{N}=16)$ & GDM2 $(\mathrm{N}=25)$ & GDM3 $(\mathrm{N}=9)$ & Control $(\mathrm{N}=25)$ & Overall $P$-value \\
\hline Gestational weight gain, $\mathrm{kg}$ & $9.1 \pm 5.0^{*}$ & $10.1 \pm 5.7^{*}$ & $13.9 \pm 7.5$ & $15.2 \pm 7.8$ & 0.026 \\
BP syst, $\mathrm{mmHg}$ & $123 \pm 16$ & $118 \pm 4$ & $127 \pm 11^{* \$}$ & $116 \pm 8$ & 0.016 \\
BP diast, $\mathrm{mmHg}$ & $79 \pm 7^{*}$ & $76 \pm 5$ & $83 \pm 9 *$ & $73 \pm 6$ & 0.002 \\
BG average, mmol/1 & $5.7 \pm 0.4$ & $5.8 \pm 0.3$ & $6.2 \pm 0.7$ & $6.0 \pm 0.5$ & 0.051 \\
Fasting BG, mmol/l & $4.8 \pm 0.3$ & $4.8 \pm 0.4$ & $5.0 \pm 0.5$ & $4.7 \pm 0.3$ & 0.592 \\
1 h postprandial BG, mmol/1 & $6.1 \pm 0.6$ & $6.1 \pm 0.4$ & $6.6 \pm 0.9$ & $6.5 \pm 0.7$ & 0.078 \\
$\%$ (N) treated with insulin & $31 \%(5)$ & $40 \%(10)$ & $33 \%(3)$ & $\mathrm{N} / \mathrm{A}$ & 0.836 \\
\hline
\end{tabular}

BG: blood glucose derived from electronic dairies filled in by participants (N GDM1 = 15, N GDM2= 24, N GDM3 = 5, N control $=8$ ) during the study period; BP: blood pressure measured within one week before delivery; N/A: non applicable.

$* P<0.05$ vs. control.

$\$ P<0.05$ vs. GDM2.

Table 3. Pregnancy outcomes and biochemical parameters in cord blood.

\begin{tabular}{llllll}
\hline \hline & GDM 1 $(\mathrm{N}=16)$ & GDM2 $(\mathrm{N}=25)$ & GDM3 (N =9) & Control (N=25) $P$-value \\
\hline Gestational age at delivery, weeks & $39.2 \pm 1.6$ & $39.2 \pm 0.9$ & $38.9 \pm 1.5$ & $39.7 \pm 1.0$ & 0.247 \\
Cesarean section, N (\%) & $25 \%(4)$ & $24 \%(6)$ & $33 \%(3)$ & $20 \%(5)$ & 0.883 \\
Birth weight, g & $3563 \pm 495$ & $3587 \pm 546$ & $3734 \pm 500$ & $3513 \pm 555$ & 0.688 \\
LGA, \% & $13 \%(2)$ & $30 \%(7)$ & $56 \%(5)$ & $12 \%(3)$ & 0.033 \\
SGA, \% (N) & $6 \%(1)$ & $9 \%(2)$ & $11 \%(1)$ & $4 \%(1)$ & 0.872 \\
C-peptide, pmol/1 & $297.9 \pm 132.4$ & $297.9 \pm 198.6$ & $430.3 \pm 231.7$ & $297.9 \pm 132.4$ & 0.258 \\
Glucose, mmol/l & $4.7 \pm 1.1$ & $5.2 \pm 1.3$ & $4.3 \pm 0.8$ & $4.5 \pm 1.2$ & 0.221 \\
Cholesterol, mmol/1 & $1.8 \pm 0.4$ & $1.8 \pm 0.4$ & $1.6 \pm 0.4$ & $1.6 \pm 0.6$ & 0.184 \\
LDL-C, mmol/l & $0.95 \pm 0.75$ & $0.89 \pm 0.46$ & $0.65 \pm 0.22$ & $0.78 \pm 0.41$ & 0.340 \\
HDL-C, mmol/l & $0.81 \pm 0.23$ & $0.73 \pm 0.32$ & $0.79 \pm 0.22$ & $0.78 \pm 0.40$ & 0.449 \\
Triglycerides, mmol/l & $0.42 \pm 0.14$ & $0.35 \pm 0.18$ & $0.29 \pm 0.12$ & $0.47 \pm 0.33$ & 0.279 \\
\hline \hline
\end{tabular}

LGA: large for gestational age; SGA: small for gestational age; HDL-C: high-density lipoprotein-cholesterol; LDL-C: lowdensity lipoprotein cholesterol.

LGA was defined by a birth weight exceeding the 90th percentile on standard charts. SGA was defined by a birth weight below the 10th percentile on standard charts.

${ }^{*} P=0.017$ compared to control group. 


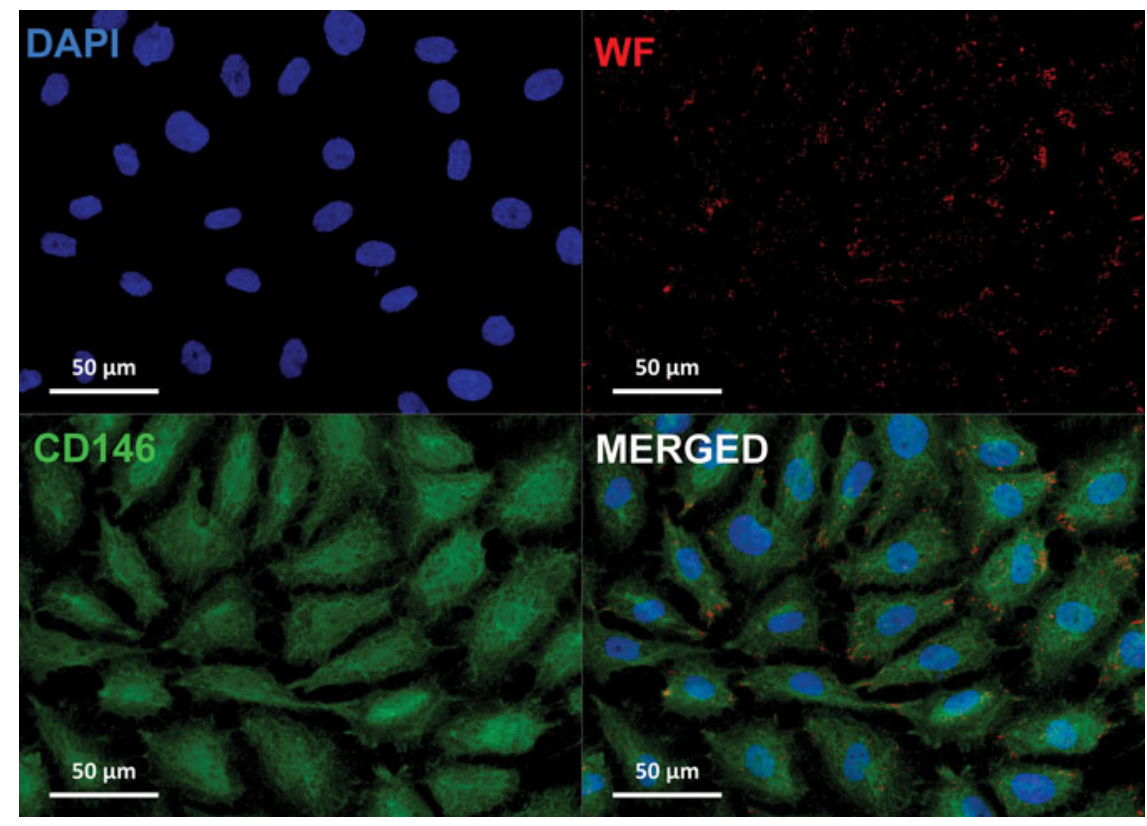

Fig. 1. Immunocytochemical staining of endothelial markers von Willebrand factor and CD146.

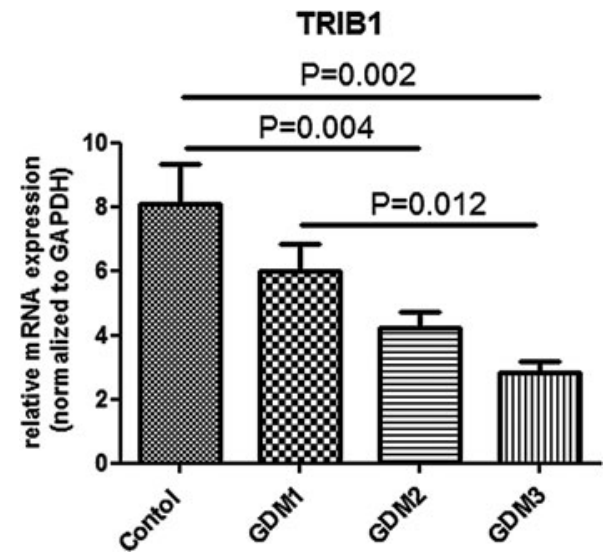

Fig. 2. TRIB1 gene expression in human umbilical vein endothelial cells.

\section{Discussion}

In our study $T R I B I$ gene expression appeared to be lower in HUVECs of newborns from two groups of GDM with late start of treatment (29-32 weeks and $\geq 34$ weeks gestation) compared to controls. Notably, the lowest level of TRIBI gene expression was observed in women with the latest start of treatment for GDM ( $>34$ weeks). The difference was gone if treatment had been started before the 28th week of gestation. The data obtained confirm the influence of intrauterine hyperglycaemia on TRIBI gene expression in HUVECs, which may potentially program predisposition to coronary artery disease (CAD) in offspring.

The fact that women with treatment started between 29-32 weeks gestation still had a decreased level of TRIB1 mRNA compared to controls, and the negative correlation of the level of TRIBI gene expression with maternal gestational age at the time when treatment of GDM started supports the importance of timely diagnosis and treatment of GDM, namely at 24-28 weeks of gestation as recommended by current guidelines (International Association of Diabetes and Pregnancy Study Groups Consensus Panel, 2010; Dedov et al., 2012), for achievement of a normal level of TRIBI gene expression. It may contribute to prevention of CAD in offspring, taking into consideration the role of the TRIBI gene in controlling lipid levels. Further research with follow-up of offspring is needed to support this hypothesis.

Additional studies comparing the levels of expression of other genes involved in CAD development derived from newborns of women with different timeframes of GDM diagnosis, including those diagnosed before the 24th week (according to some guidelines [International Association of Diabetes and Pregnancy Study Groups Consensus Panel, 2010; Dedov et al., 2012]) would be of particular interest. However, doing an OGTT too early may miss women with GDM, as hyperglycaemia may develop later. GDM is usually diagnosed between the 24th and 28th week of pregnancy, which is the moment when maternal insulin resistance rises to derive the transfer of nutrients towards the fetus. Nevertheless, there is evidence that GDM may develop even after the 30th week (Kurtbas et al., 2011).

As fasting, postprandial and average glucose levels achieved during the study were similar among GDM groups and controls, we presume that the women with GDM were treated effectively. 
It should be pointed out that the results of our study were obtained in the environment of mild hyperglycaemia in the mother. Although the women included in this study were diagnosed with GDM based on the IADPSG criteria (International Association of Diabetes and Pregnancy Study Groups Consensus Panel, 2010), only 11 women met the Carpenter and Coustan criteria which classify more severe cases of hyperglycaemia during pregnancy as GDM (Carpenter \& Coustan, 1982).

We found that TRIBI gene expression in HUVECs was potentially affected by GDM exposure. However, there could be some other causes for the observed changes.

Apart from the difference in the levels of glucose, the studied groups also had several other differences in clinical parameters that could be associated with TRIB1 gene expression, namely, systolic and diastolic $\mathrm{BP}$, serum levels of lipids and different weight gain during pregnancy. For BP and lipid levels, it should be taken into consideration that they were measured at higher gestational age (after the 34th week) in the GDM3 group. It is known that both parameters normally increase as pregnancy progresses (Pusukuru et al., 2016; Mikami et al., 2017). So, the difference in these parameters could result from different gestational age at the time of their measurement. The difference in these parameters among the groups could also be a result of the absence of treatment before the 34th week, as GDM is a well known risk factor for gestational hypertensive disorders (HAPO Study Cooperative Research Group et al., 2008) and treatment of GDM reduces the risk of preeclampsia and reduces lipid levels (Landon et al., 2009). However, as we do not have blood samples stored at the time of OGTT, we cannot exclude the possibility that it was a baseline difference among the groups. Though it is unlikely, taking into consideration the absence of difference in the age and prepregnancy BMI. As for the difference in gestational weight gain between GDM1, GDM2 and the respective control group, the influence of weight gain on TRIB1 gene expression is contradicted by the fact that the weight gain was comparable in the GDM3 and control groups while there was the most prominent difference in TRIBI gene expression between these groups.

The absence of correlation of TRIB1 mRNA levels with gestational weight gain also does not support the hypothesis that gestational weight gain may influence TRIB1 expression.

Another plausible factor that could influence the results was the type of treatment of the GDM mothers (diet vs. diet + insulin). However, there was no correlation of TRIB1 mRNA levels with insulin treatment. This finding is in line with the data from the El Hajj et al., study, where no DNA methylation difference between the diet and diet + insulin treatment groups was found (El Hajj et al., 2013). Of interest, a recent study by Koning et al., has shown no difference in clinical outcomes, including LGA neonates, in women with GDM treated with diet only or with additional insulin (Koning et al., 2016).

We are not aware of any other publications addressing expression of TRIB1 in any tissues of newborns of women with GDM. However, our findings are in line with the data by Ruchat et al. (2013) who found TRIB1 among genes differentially methylated between samples either exposed to GDM or not.

In a recent study by Amrithraj et al. (2017), HUVECs derived from newborns of women with GDM exerted a lower proliferation rate compared with the normal control group. In our cohort we did not observe such a difference. This discrepancy may be explained by the difference in the severity of maternal hyperglycaemia between the studies. Amrithraj et al. (2017) selected five severe GDM cases from their specimen collection based on high OGTT values, while our study included women with relatively mild hyperglycaemia as diagnosed by IASDPG criteria.

Despite the difference in the levels of TRIB1 expression we did not reveal a difference in the cord blood serum lipid levels. Maybe the changes in lipid levels due to decreased TRIB1 activity will appear later in the offspring. Follow-up of the children born to the participants of the study will address this issue and the question of whether TRIB1 expression is altered in other tissues (e.g., peripheral blood leucocytes) and if this alteration is stable over a long period of time.

As for the maternal lipid levels, the fact that women from the GDM3 group had lower levels of HDL-C compared to all other studied groups may be a result of a more advanced gestational age at the time of analysis, as HDL-C is known to decrease with progression of pregnancy (Pusukuru et al., 2016). However, we cannot exclude the baseline difference in HDL-C levels because we do not have blood samples collected at the same gestational ages.

Another interesting finding is the negative correlation of the level of TRIB1 gene expression with the SGA state of the newborns. This finding supports the well known role of low birth weight in fetal metabolic programming including predisposition to $\mathrm{CAD}$ (Barker, 1999) and suggests that it could also be mediated through the alteration of TRIBI gene expression.

The limitations of our study are the observational design, the small sample size, especially of the GDM3 group, and the absence of blood samples from this group at the time of OGTT.

However, due to ethical considerations, it is impossible to avoid this limitation and organize a randomized trial with one of the arms untreated until the end of the third trimester. Thus, our study including 
groups of women diagnosed with GDM at different gestational ages up to one week before delivery, though small, provides a unique opportunity to look at the mechanisms underlying the influence of prolonged intrauterine hyperglycaemia on fetal developmental programming.

The study was funded by Russian Science Foundation (project No.15-14-30012).

\section{Declaration of interest}

None.

\section{References}

Amrithraj, A. I., Kodali, A., Nguyen, L., Teo, A. K. K., Chang, C. W., Karnani, N., Ng, K. L., Gluckman, P. D., Chong, Y. S. \& Stünkel, W. (2017). Gestational diabetes alters functions in offspring's umbilical cord cells with implications for cardiovascular health. Endocrinology 158(7), 2102-2112.

Angyal, A. \& Kiss-Toth, E. (2012). The tribbles gene family and lipoprotein metabolism. Current Opinion in Lipidology 23(2), 122-126.

Barker, D. J. (1999). Fetal origins of cardiovascular disease. Annals of Medicine 31(Suppl. 1), 3-6.

Bouchard, L., Hivert, M.F., Guay, S. P., St-Pierre, J., Perron, P. \& Brisson, D. (2012). Placental adiponectin gene DNA methylation levels are associated with mothers' blood glucose concentration. Diabetes 61(5), $1272-1280$.

Bouchard, L., Thibault, S., Guay, S. P., Santure, M., Monpetit, A., St-Pierre, J., Perron, P. \& Brisson, D. (2010). Leptin gene epigenetic adaptation to impaired glucose metabolism during pregnancy. Diabetes Care 33(11), 2436-2441.

Burkhardt, R., Toh, S. A., Lagor, W. R., Birkeland, A., Levin, M., Li, X., Robblee, M., Fedorov, V. D., Yamamoto, M., Satoh, T., Akira, S., Kathiresan, S., Breslow, J. L. \& Rader, D. J. (2010). Tribl is a lipidand myocardial infarction-associated gene that regulates hepatic lipogenesis and VLDL production in mice. Journal of Clinical Investigation 120, 4410-4414.

Carpenter, M. W. \& Coustan, D. R. (1982). Criteria for screening tests for gestational diabetes. Americal Journal of Obstetrics and Gynecology 144, 768-773.

Damm, P., Houshmand-Oeregaard, A., Kelstrup, L., Lauenborg, J., Mathiesen, E. R. \& Clausen, T. D. (2016). Gestational diabetes mellitus and long-term consequences for mother and offspring: a view from Denmark. Diabetologia 59(7), 1396-1399.

Dedov, I. I., Krasnopol'skiy, V. I. \& Sukhikhm, G. T. (2012). Russian National Consensus Statement on gestational diabetes: diagnostics, treatment and postnatal care. Diabetes Mellitus 4, 4-10.

Edmondson, A. C., Braund, P. S., Stylianou, I. M., Khera, A. V., Nelson, C. P., Wolfe, M. L., Derohannessian, S. L., Keating, B. J., Qu, L., He, J., Tobin, M. D., Tomaszewski, M., Baumert, J., Klopp, N., Döring, A., Thorand, B., Li, M., Reilly, M. P., Koenig, W., Samani, N. J. \& Rader, D. J. (2011). Dense genotyping of candidate gene loci identifies variants associated with high-density lipoprotein cholesterol. Circulation. Cardiovascular Genetics 4, 145-155.

El Hajj, N., Pliushch, G., Schneider, E., Dittrich, M., Müller, T., Korenkov, M., Aretz, M., Zechner, U., Lehnen, H. \& Haaf, T. (2013). Metabolic programming of MEST DNA methylation by intrauterine exposure to gestational diabetes mellitus. Diabetes 62, 1320-1328.

HAPO Study Cooperative Research Group, Metzger, B. E., Lowe, L. P., Dyer, A. R., Trimble, E. R., Chaovarindr, U., Coustan, D. R., Hadden, D. R., McCance, D. R., Hod, M., McIntyre, H. D., Oats, J. J., Persson, B., Rogers, M.S. \& Sacks, D. A. (2008). Hyperglycaemia and adverse pregnancy outcomes. New England Journal of Medicine 358, 1991-2002.

International Association of Diabetes and Pregnancy Study Groups Consensus Panel (2010). International Association of Diabetes and Pregnancy Study Groups recommendations on the diagnosis and classification of hyperglycaemia in pregnancy. Diabetes Care 33, 676-682.

Jaffe, E. A., Nachman, R. L., Becker, C. G. \& Minick, C. R. (1973). Culture of human endothelial cells derived from umbilical veins. Journal of Clinical Investigation 52, $2745-2756$

Koning, S. H., Hoogenberg, K., Scheuneman, K. A., Baas, M. G., Korteweg, F. J., Sollie, K. M., Schering, B. J., van Loon, A. J., Wolffenbuttel, B. H., van den Berg, P. P. \& Lutgers, H. L. (2016). Neonatal and obstetric outcomes in diet- and insulin-treated women with gestational diabetes mellitus: a retrospective study. BMC Endocrine Disorders 16, 52.

Kurtbas, H., Keskin, H. L. \& Avsar, A. F. (2011). Effectiveness of screening for gestational diabetes during the late gestational period among pregnant Turkish women. Journal of Obstetric and Gynaecology Research 37, 520-526.

Landon, M. B., Spong, C. Y., Thom, E., Carpenter, M. W., Ramin, S. M., Casey, B., Wapner, R. J., Varner, M. W., Rouse, D. J., Thorp, J. M. Jr., Sciscione, A., Catalano, P., Harper, M., Saade, G., Lain, K. Y., Sorokin, Y., Peaceman, A. M., Tolosa, J.E., Anderson, G. B. \& Eunice Kennedy Shriver National Institute of Child Health and Human Development Maternal-Fetal Medicine Units Network (2009). A multicenter, randomised trial of treatment for mild gestational diabetes. New England Journal of Medicine 361, 1339-1348.

Livak, K. J. \& Schmittgen, T. D. (2001). Analysis of relative gene expression data using real-time quantitative PCR and the 2(-Delta Delta C(T)) Method. Methods 25, 402-408.

Ma, R. C., Tutino, G. E., Lillycrop, K. A., Hanson, M. A. \& Tam, W. H. (2015). Maternal diabetes, gestational diabetes and the role of epigenetics in their long term effects on offspring. Progress in Biophysics and Molecular Biology 118(1-2), 55-68.

Mikami, Y., Takai, Y., Era, S., Ono, Y., Saitoh, M., Baba, K., Suzuki, H. \& Seki, H. (2017) Provisional criteria for the diagnosis of hypertension in pregnancy using home blood pressure measurements. Hypertension Research $\mathbf{4 0}$ (7), 679-684.

Nakayama, K., Bayasgalan, T., Yamanaka, K., Kumada, M., Gotoh, T., Utsumi, N., Yanagisawa, Y., Okayama, M., Kajii, E., Ishibashi, S., Iwamoto, S. \& Jichi Community Genetics Team (JCOG) (2009). Large scale replication analysis of loci associated with lipid concentrations in a Japanese population. Journal of Medical Genetics 46, 370-374.

Nielsen, G. L., Dethlefsen, C., Lundbye-Christensen, S., Pedersen, J. F., Mølsted-Pedersen, L. \& Gillman, M. W. (2012). Adiposity in 277 young adult male offspring of 
women with diabetes compared with controls: a Danish population-based cohort study. Acta Obstetricia et Gynecologica Scandinavica 91(7), 838-843.

Pettitt, D. J., Lawrence, J. M., Beyer, J., Hillier, T. A., Liese, A. D., Mayer-Davis, B., Loots, B., Imperatore, G., Liu, L., Dolan, L. M., Linder, B. \& Dabelea, D. (2008). Association between maternal diabetes in utero and age at offspring's diagnosis of type 2 diabetes. Diabetes Care 31(11), 2126-2130.

Pusukuru, R., Shenoi, A. S., Kyada, P. K., Ghodke, B., Mehta, V., Bhuta, K. \& Bhatia, A. (2016). Evaluation of lipid profile in second and third trimester of pregnancy. Journal of Clinical and Diagnostic Research 10(3), QC12QC16.

Ruchat, S.-M., Houde, A. A., Voisin, G., St-Pierre, J., Perron, P., Baillargeon, J.-P., Gaudet, D., Hivert, M. F., Brisson, D. \& Bouchard, L. (2013). Gestational diabetes mellitus epigenetically affects genes predominantly involved in metabolic diseases. Epigenetics 8(9), 935-943.

Sacks, A. D., Hadden, R. D., Maresh, M., Deerochanawong, C., Dyer, R. A., Metzger, B. E., Lowe, L. P., Coustan, D. R., Hod, M., Oats, J. M., Persson, B. \& Trimble, E. R. (2012) Frequency of gestational diabetes mellitus at collaborating centers based on IADPSG Consensus. Diabetes Care 35, 526-528.

Tai, E. S., Sim, X. L., Ong, T. H., Wong, T. Y., Saw, S. M., Aung, T., Kathiresan, S., Orho-Melander, M., Ordovas, J. M., Tan, J. T. \& Seielstad, M. (2009). Polymorphisms at newly identified lipid-associated loci are associated with blood lipids and cardiovascular disease in an
Asian Malay population. Journal of Lipid Research $\mathbf{5 0}$, 514-520.

Tam, W. H., Ma, R.C., Yang, X., Li, A. M., Ko, G. T., Kong, A.P., Lao, T. T., Chan, J.C., Lam, C. W. \& Chan, J. C. (2010). Glucose intolerance and cardiometabolic risk in adolescents exposed to maternal gestational diabetes: a 15-year follow-up study. Diabetes Care 33(6), $1382-1384$.

Varbo, A., Benn, M., Tybjaerg-Hansen, A., Grande, P. \& Nordestgaard, B. G. (2010). TRIB1 and GCKR polymorphisms, lipid levels, and risk of ischemic heart disease in the general population. Arteriosclerosis, Thrombosis, and Vascular Biology 31, 451-457.

Willer, C. J., Sanna, S., Jackson, A. U., Scuteri, A., Bonnycastle, L. L., Clarke, R., Heath, S. C., Timpson, N. J., Najjar, S. S., Stringham, H. M., Strait, J., Duren, W. L., Maschio, A., Busonero, F., Mulas, A., Albai, G., Swift, A. J., Morken, M. A., Narisu, N., Bennett, D., Parish, S., Shen, H., Galan, P., Meneton, P., Hercberg, S., Zelenika, D., Chen, W. M., Li, Y., Scott, L. J., Scheet, P. A., Sundvall, J., Watanabe, R. M., Nagaraja, R., Ebrahim, S., Lawlor, D. A., Ben-Shlomo, Y., Davey-Smith, G., Shuldiner, A. R., Collins, R., Bergman, R. N., Uda, M., Tuomilehto, J., Cao, A., Collins, F. S., Lakatta, E., Lathrop, G. M., Boehnke, M., Schlessinger, D., Mohlke, K. L. \& Abecasis, G. R (2008). Newly identified loci that influence lipid concentrations and risk of coronary artery disease. Nature Genetics 40(2), 161-169. 